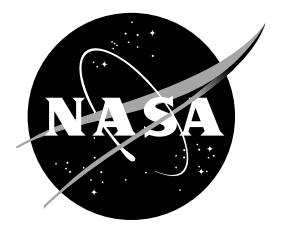

\title{
Developing Architectures and Technologies for an Evolvable NASA Space Communication Infrastructure
}

Kul Bhasin

Glenn Research Center, Cleveland, Ohio

Jeffrey Hayden

Infinite Global Infrastructures LLC, West Chicago, Illinois 
Since its founding, NASA has been dedicated to the advancement of aeronautics and space science. The NASA Scientific and Technical Information (STI) Program Office plays a key part in helping NASA maintain this important role.

The NASA STI Program Office is operated by Langley Research Center, the Lead Center for NASA's scientific and technical information. The NASA STI Program Office provides access to the NASA STI Database, the largest collection of aeronautical and space science STI in the world. The Program Office is also NASA's institutional mechanism for disseminating the results of its research and development activities. These results are published by NASA in the NASA STI Report Series, which includes the following report types:

- $\quad$ TECHNICAL PUBLICATION. Reports of completed research or a major significant phase of research that present the results of NASA programs and include extensive data or theoretical analysis. Includes compilations of significant scientific and technical data and information deemed to be of continuing reference value. NASA's counterpart of peerreviewed formal professional papers but has less stringent limitations on manuscript length and extent of graphic presentations.

- TECHNICAL MEMORANDUM. Scientific and technical findings that are preliminary or of specialized interest, e.g., quick release reports, working papers, and bibliographies that contain minimal annotation. Does not contain extensive analysis.

- CONTRACTOR REPORT. Scientific and technical findings by NASA-sponsored contractors and grantees.
- CONFERENCE PUBLICATION. Collected papers from scientific and technical conferences, symposia, seminars, or other meetings sponsored or cosponsored by NASA.

- SPECIAL PUBLICATION. Scientific, technical, or historical information from NASA programs, projects, and missions, often concerned with subjects having substantial public interest.

- TECHNICAL TRANSLATION. Englishlanguage translations of foreign scientific and technical material pertinent to NASA's mission.

Specialized services that complement the STI Program Office's diverse offerings include creating custom thesauri, building customized databases, organizing and publishing research results ... even providing videos.

For more information about the NASA STI Program Office, see the following:

- Access the NASA STI Program Home Page at http://www.sti.nasa.gov

- E-mail your question via the Internet to help@sti.nasa.gov

- Fax your question to the NASA Access Help Desk at 301-621-0134

- Telephone the NASA Access Help Desk at 301-621-0390

- Write to:

NASA Access Help Desk

NASA Center for AeroSpace Information 7121 Standard Drive

Hanover, MD 21076 
NASA/TM-2004-213108

AIAA-2004-3253

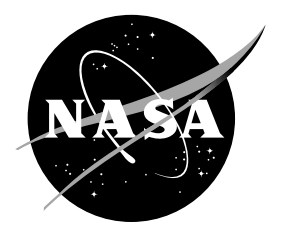

\section{Developing Architectures and Technologies for an Evolvable NASA Space Communication Infrastructure}

Kul Bhasin

Glenn Research Center, Cleveland, Ohio

Jeffrey Hayden

Infinite Global Infrastructues LLC, West Chicago, Illinois

Prepared for the

22nd International Communications Satellite Systems Conference and Exhibit 2004 (ICSSC) sponsored by the American Institute of Aeronautics and Astronautics

Monterey, California, May 9-12, 2004

National Aeronautics and

Space Administration

Glenn Research Center 


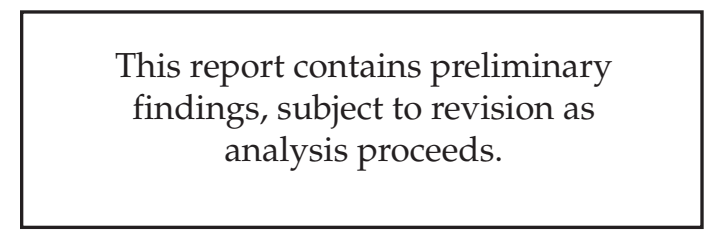

$\begin{array}{lr} & \text { Available from } \\ \text { NASA Center for Aerospace Information } & \text { National Technical Information Service } \\ \text { 7121 Standard Drive } & 5285 \text { Port Royal Road } \\ \text { Hanover, MD 21076 } & \text { Springfield, VA 22100 }\end{array}$

Available electronically at http://gltrs.grc.nasa.gov 


\title{
Developing Architectures and Technologies for an Evolvable NASA Space Communication Infrastructure
}

\author{
Kul Bhasin \\ National Aeronautics and Space Administration \\ Glenn Research Center \\ Cleveland, Ohio 44135 \\ Jeffrey L. Hayden \\ Infinite Global Infrastructures LLC \\ West Chicago, Illinois 60185
}

\begin{abstract}
Space communications architecture concepts play a key role in the development and deployment of NASA's future exploration and science missions. Once a mission is deployed, the communication link to the user needs to provide maximum information delivery and flexibility to handle the expected large and complex data sets and to enable direct interaction with the spacecraft and experiments. In human and robotic missions, communication systems need to offer maximum reliability with robust two-way links for software uploads and virtual interactions. Identifying the capabilities to cost effectively meet the demanding space communication needs of $21^{\text {st }}$ century missions, proper formulation of the requirements for these missions, and identifying the early technology developments that will be needed can only be resolved with architecture design. This paper will describe the development of evolvable space communication architecture models and the technologies needed to support Earth sensor web and collaborative observation formation missions; robotic scientific missions for detailed investigation of planets, moons, and small bodies in the solar system; human missions for exploration of the Moon, Mars, Ganymede, Callisto, and asteroids; human settlements in space, on the Moon, and on Mars; and great in-space observatories for observing other star systems and the universe. The resulting architectures will enable the reliable, multipoint, high data rate capabilities needed on demand to provide continuous, maximum coverage of areas of concentrated activities, such as in the vicinity of outposts inspace, on the Moon or on Mars.
\end{abstract}

\section{Introduction}

Space communications architectures and technologies in the 21 st century must meet the growing needs of Earth sensor web and collaborative observation formation missions, robotic scientific missions for detailed investigation of planets, moons, and small bodies in the solar system; human missions for exploration of the Moon, Mars, Ganymede, Callisto, and asteroids; human settlements in space, on the Moon, and on Mars; and great in-space observatories for observing other star systems and the universe ${ }^{1}$. An advanced, integrated, communications infrastructure will enable the reliable, multipoint, high data rate capabilities needed on demand to provide continuous, maximum coverage of areas of concentrated activities, such as in the vicinity of in-space outposts, the Moon or Mars.

Past work in space communications was developed from several unrelated perspectives of the different enterprises with a view toward providing communication services for each new mission as it came along. Communications for Earth observing missions ${ }^{2}$, for instance, were developed independently from what was needed for other missions, such as the human shuttle and ISS missions. NASA implemented the Tracking and Data Relay Satellite System (TDRSS) as a space network for general use by NASA's human missions and Earth observing missions. Unfortunately, the costs of using the TDRSS services (special on-board communication equipment, the TDRSS White Sands Complex, and dedicated ground networks) were considered too high for most Earth observing missions, so those missions used new and modified older ground stations for capturing their data. Communications for Mars and deep space missions also developed independently from the others and shared the use of the Deep 
Space Network (DSN). Communications were again treated from a services perspective ${ }^{3}$, and although the interfaces and protocols used for different missions were standardized, the standards could not support autonomous networking and data routing. More recently, the enterprises have been accumulating the capabilities that are felt to be necessary for future missions. However, the enterprise solutions identified for future communications remain services-centric, that is, the solutions are specific to each enterprise's missions and are not integrated into an overall NASA communication infrastructure solution, wherein the in-space nodes can communicate with each other as well as with users on Earth through the Internet. The commercial Iridium communication satellite constellation, although not as successful as originally anticipated, did prove that inter-spacecraft communications and networking was possible.

The approach taken in this paper is architecture-centric in that the work will lead to an integrated, internetworked, space communications infrastructure developed from architectural elements and interfaces. Within this networked infrastructure, data will move from sensor to user under autonomous control of the nodes within the network. Human operations will become maintenance and network administrative functions. To obtain the requirements that follow, node-to-node link capability needs were captured from data provided by the enterprise mission planners and technologists. These capabilities include data rates, distance, and function needed over each general link from the Earth-side network and terminal to the in-space user node. Later work will extend into defining and standardizing the hardware and software interfaces to be implemented in each node and identifying the most appropriate technologies to implement for those nodes. It is expected that this architectural development work will need to continue as the infrastructure is first emplaced and then as it grows with time.

In this paper, we describe an integrated communications architecture that will support the Vision for Space Exploration articulated by President Bush on January 16, 2004'; we provide a summary of the communications needs and capabilities that the nodes in the resulting new infrastructure will satisfy; and we then identify the architectural tradeoffs and the technology gaps that must be resolved to achieve a workable new architecture. We discuss those elements of the communications infrastructure that enable and enhance robotic and human exploration of the Moon and Mars. We do not cover communication support for robotic missions to the outer planets.

\section{Space Communication Architecting Process}

The overall space communication architecture shown in Fig. 1 was developed based on NASA's needs and requirements collected through participative processes ${ }^{4}$. This paper takes a first look at the space communication architecture in an integrated fashion while addressing the needs of the NASA enterprises. The figure shows the scenario of a networked space communications infrastructure with connections to the regions of interest within the solar system ${ }^{5}$. The communication capabilities are provided by constellations of communications relay satellites; sensor web inter-spacecraft communications packages for relaying data between science observation satellites in high Earth orbits; high data rate, small, autonomous ground terminals; communications relay spacecraft placed in gravitationally balanced Lagrange orbits between the Earth and Moon and the Earth and sun; relay satellites around the Moon; and science and relay satellites placed in orbit around Mars, the outer planets and small bodies. The communication links shown in Fig. 1 are further described in the following sections.

\section{A. Architecture Elements and Interfaces}

The architecture is represented by four architectural elements ${ }^{4}$. Blue lines indicate high rate, inter-nodal links between backbone elements; red lines are links from access elements (i.e., robotic and human exploration spacecraft) to backbone elements; green lines are inter-spacecraft links; and yellow lines indicate short range between proximity elements. Collectively, links within and between these elements represent segments of the pathways needed to achieve the end-to-end data-passing capability envisioned for future NASA communications. The high rate backbone network elements are the intra-network structures of high rate communication nodes and inter-nodal links that utilize advanced communication technologies to increase data rate by orders of magnitude while reducing overall costs. The flexible access network elements are re-configurable communication systems at the edges of the backbone networks that enable in-space humans, robotic spacecraft, aircraft, or ground vehicles to communicate to the infrastructure edge-nodes. Inter-spacecraft cooperative network elements incorporate the technologies necessary to enable intercommunications between future NASA spacecraft flying in formation, in clusters, or in constellations. Proximity wireless network elements include: short range, low power, low cost communications packages for inter-communication between small sensor packages, and small wireless local area network (WLAN) packages to support high data rate, bidirectional communications for voice, video, data, and control between humans and robots over a distance of meters to a few kilometers. 


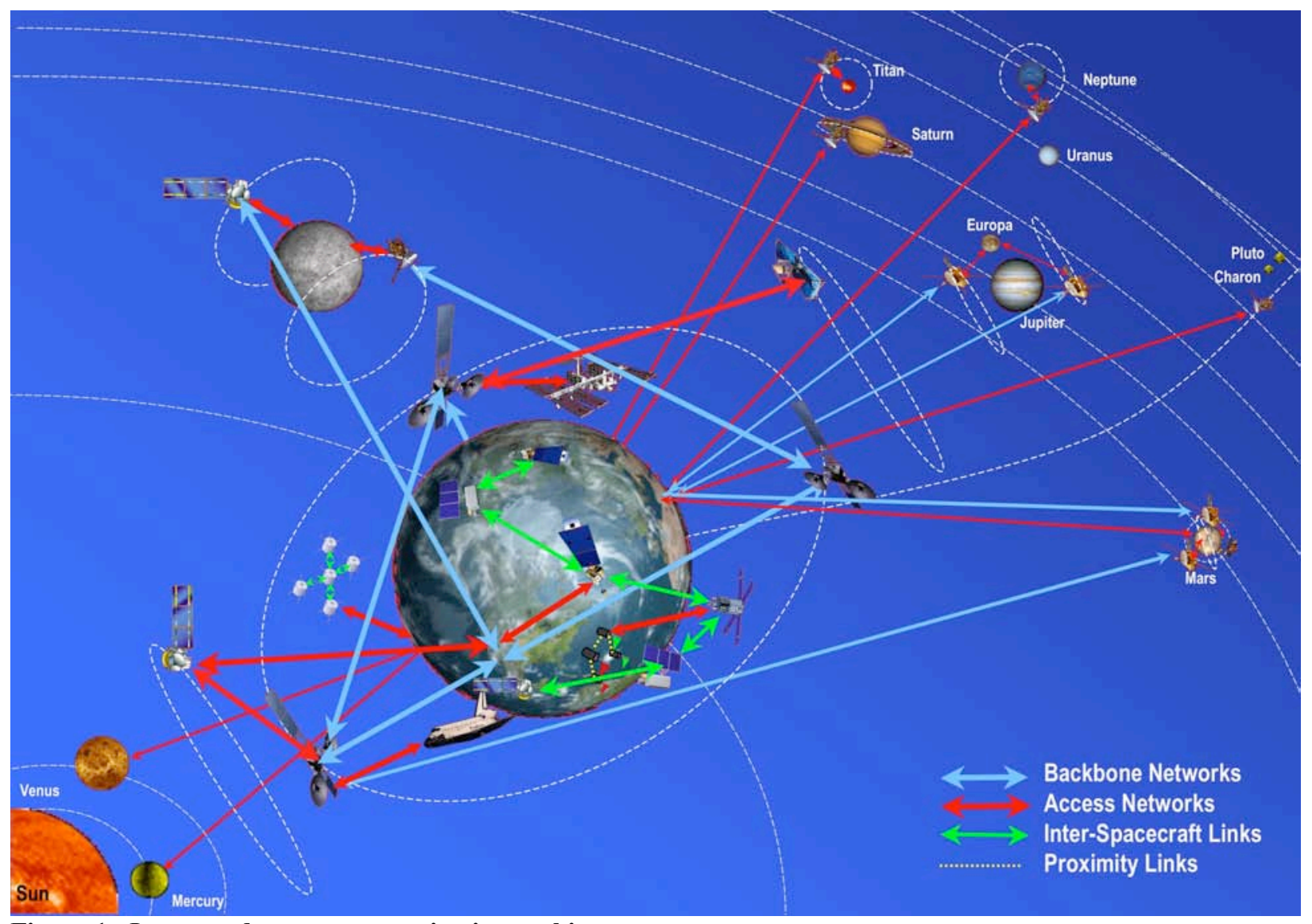

Figure 1: Integrated space communication architecture.

\section{B. Layered/Integrated Communications Architecture}

With integrated architectures, NASA will be able to achieve intelligent communications. The communication networking paths will utilize the lower five of the seven Open System Interconnection (OSI) model layers (Fig. 2) to

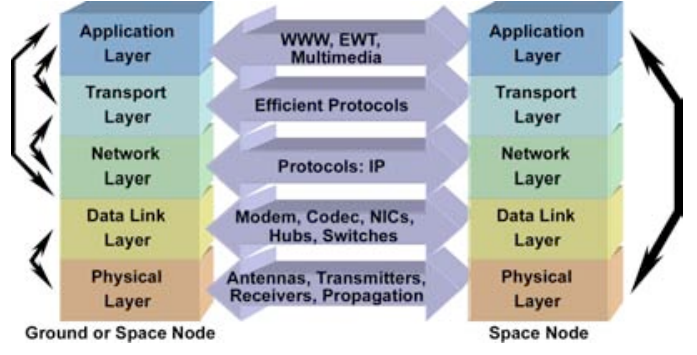

Figure 2:Internet protocol layers used in integrated communication architecture. achieve Internet protocol (IP) data routing capabilities ${ }^{6}$. Current approaches have only nominal interaction between these layers ${ }^{7}$. However, interactive control between the layers enables autonomous data routing on-board and between spacecraft by allowing control of antenna pointing, transmitter power, transmit data rates and media access methods that vary with distance, thus permitting a complete end-to-end data routing capability. It also enables spacecraft or users to demand access to the network as if they were making a cellular phone call. Common protocols and interfaces at these layers will enable inter-active links to be made and broken on demand of any node in the network, thus enabling complex and deeply networked communications channels between nodes in space and on Earth.

\section{Communication Nodes - Descriptions and Options}

As the next step, the individual communication nodes within each region of the evolutionary architecture model were identified ${ }^{5}$. These nodes included all entities (sensors, spacecraft, aircraft, robots, humans, etc.) that might communicate with each other inside or outside of the region. Then links for each pair of nodes that might reasonably be expected to inter-communicate were identified. This provided a view of all the links into and out of a particular node and a means to tabulate their physical and desired characteristics. These node-to-node links become the optional building blocks of the architectures. There are multiple paths by which data can move from one node to 
another. The existence of a path depends on whether a particular architectural element option is chosen for implementation into the infrastructure. Many node-to-node link options will likely drop out of consideration with further analysis.

The Earth vicinity communications nodal group encompasses the communications infrastructure needed to support robotic and human missions from the Earth surface to high Earth orbit (HEO). It includes: that part of the DoD's transformational communications architecture (TCA) (Armstrong, 2003) that NASA may implement and/or use; communication relay satellite networks that may optionally be placed in geosynchronous Earth orbits (GEO); or high inclination Molniya orbits, medium Earth orbit (MEO), and low Earth orbit (LEO) Earth observer satellite data and command paths. The Moon vicinity nodal group encompasses the surface and orbits of the Moon and the EarthMoon system's Lagrange points. Elements of the physical communications infrastructure considered in this group include: communication relay satellites in Earth-Moon Lagrange orbit or Moon orbit, long-link Moon-to-Earth communications and wireless local area networks (WLANs) on the surface of the Moon. The Earth-Sun Lagrangian vicinity nodal group comprises those elements of the communications infrastructure that might be placed at the Earth-Sun Lagrange points L1, L2, L4, and/or L5 to provide high data rate backbone capabilities for Earth, Sun, galaxy, or universe observing missions and deep space science missions. The Mars vicinity nodal group encompasses communications infrastructure that might be implemented to support robotic and human missions at Mars. It includes: a relay satellite network for Mars that might optionally be placed in Mars synchronous orbit (MSO), Mars high orbit (MHO), and/or Mars low orbit (MLO); networks for Mars orbit, air, and surface robotic missions; and Mars human outpost communication networks. The deep space communications nodal group is the communications infrastructure that is dispersed among the outer planets and moons in support of robotic and later human missions. It includes outer planet mission communication systems and communication relay spacecraft that might be placed in Jupiter-Sun L1, L2 halo orbits.

\section{Requirements}

The high level mission communication data rate requirements in Table 1 and the required characteristics that follow motivate the need for a set of links between nodes of NASA's future space architecture. These capabilities are addressed by examining individual node-to-node links. The resulting architecture can then be used to identify and focus technology development needed to support the physical network of communications links. Once the new technologies are in place in the physical architecture, the required high-level capabilities will be fully realized.

Table 1: Infrastructure requirements.

\begin{tabular}{|c|c|c|c|c|}
\hline Nodal Group & Node to Earth & Current & 2010 & $2020+$ \\
\hline \multirow[t]{4}{*}{ Earth Vicinity } & LEO Spacecraft (Direct Link) & $150 \mathrm{Mbps}$ & $>1$ Gbps gateway, 1 Gbps D/L & 10 Gbps \\
\hline & GEO Spacecraft (Direct Link) & $150 \mathrm{Mbps}$ & $>1 \mathrm{Gbps}$ & $10 \mathrm{Gbps}$ \\
\hline & STS & $50 \mathrm{Mbps}$ & $50 \mathrm{Mbps}$ & $50 \mathrm{Mbps}$ \\
\hline & ISS & $48 \mathrm{Mbps}$ & 150 Mbps (2005) & $300 \mathrm{Mbps}$ \\
\hline Moon & $\begin{array}{l}\text { Earth-Moon L1, L2 } \\
\text { Moon }\end{array}$ & & & $\begin{array}{l}0.2 \text { up/1 down Gbps } \\
0.2 \text { up/1 down Gbps }\end{array}$ \\
\hline Earth-Sun L1, L2 & GEO relay and Earth & & $20 \mathrm{Mbps}$ & $>100 \mathrm{Mbps}$ \\
\hline \multirow[t]{3}{*}{ Mars } & Mars Science & $100 \mathrm{Kbps}$ & $5 \mathrm{Mbps}$ & 20 up/100 down Mbps \\
\hline & Mars Exploration & - & $10 \mathrm{Mbps}$ & 20 up/100 down Mbps \\
\hline & Mars Proximity Link & - & - & $1-100 \mathrm{Mbps}$ \\
\hline Outer Planets & Jupiter to Outer Heliosphere & $10 \mathrm{Kbps}$ & $1 \mathrm{Mbps}$ & $>10 \mathrm{Mbps}$ \\
\hline
\end{tabular}

\section{A. NASA Enterprises Needs}

NASA's communications infrastructure must support all varieties of science and human exploration in the future. The science to be supported ranges from observation of the Earth, Moon, Mars, and the outer planet systems to the universe. The science also includes that which is obtained during human exploration and inhabitation of space, the Moon, Mars, and outer planet moons. Most of the NASA science missions that are under study require highbandwidth communications, including (in very short summary): hyperspectral imagery, synthetic aperture radar imagery, atmospheric measurements, and radar sounding of the Earth, planets, and moons; astronomical imagery from radio frequencies to gamma rays of other star systems, the galaxy, and universe; robotic measurements of planet/moon surface and atmospheric properties; and the search for life by many means. 


\section{B. Emerging Needs for the Space Exploration Initiative}

Future robotic missions will need to operate autonomously by sensing the area around them so they can make decisions about where to go, what samples to measure, what data to report, and how to request and connect to the space communication network. Other robotic entities must be intimately connected to human operators via wireless systems that enable real-time, or delayed-time video and control for close coordination such as in assembling large space structures. The goal of the new infrastructure design is to become a space Internet that is as autonomous as possible in operation and one in which connections are made and broken as needed by the requesting entity. This kind of communications infrastructure is needed to enable access on the demand of any mission entity, including spacecraft, surface robot, in-space exploring human, and Earth user, while using as few human operators as possible to provide the capabilities. This Internet architecture also serves the needs of the public by allowing direct viewing of mission activities and enabling safe (protected against unauthorized operations) public participation in those activities.

\section{Architecture and Technology Framework for Evolvable Space Infrastructure}

Rather than change in the independent, mission-specific way that the present NASA communications infrastructure grew to support the exploratory missions of the past, the infrastructure of the future will grow in an integrated fashion and evolve to support $>100 \mathrm{Mbps}$ data rates for robotic missions to the Moon by 2010 and human missions to the Moon by 2015. Likewise, communications networks will reach 100Mbps to support robotic missions to Mars by 2015 and human missions beyond 2020. The characteristics required by the evolving infrastructure are shown in Table 2.

Table 2: Required characteristics of the infrastructure.

\begin{tabular}{|c|c|}
\hline Required Characteristic & Rationale \\
\hline Be available $24 / 7$ & Basic requirement of human missions and most missions requiring low latency data return. \\
\hline Integrated Architectures & $\begin{array}{l}\text { Use of standard interfaces (hardware, wireless, and protocols) across the infrastructure increases data } \\
\text { routing options and reduces costs of implementation. }\end{array}$ \\
\hline $\begin{array}{l}\text { Low cost, modular and } \\
\text { efficient. }\end{array}$ & This can be achieved by adapting commercial technology standards to use in space. \\
\hline $\begin{array}{l}\text { Handle multipoint connections } \\
\text { to multiple nodes } \\
\text { simultaneously. }\end{array}$ & $\begin{array}{l}\text { Essential for broadcasting data to many spacecraft simultaneously; for inter-spacecraft coordination of } \\
\text { timing, maneuvers, and collaborative science data gathering; and for enabling autonomous end-to-end } \\
\text { routing of data. }\end{array}$ \\
\hline Highly reliable connections & $\begin{array}{l}\text { Connections must be reliable to meet the very high data rates or the required characteristics will not be } \\
\text { met. }\end{array}$ \\
\hline Long life expectancy. & High cost of development and space flight dictates lifetimes of greater than 20 years. \\
\hline Highly reconfigurable & To accommodate upgrades and enable growth in capabilities over time. \\
\hline Be secure. & Cannot allow intruders to take control of the systems nor allow sampling of private data. \\
\hline Connect End-to-end & $\begin{array}{l}\text { Enabling data to move on demand from user to spacecraft instrument or back greatly reduces } \\
\text { operations support costs. }\end{array}$ \\
\hline $\begin{array}{l}\text { Handle multiple robotic and } \\
\text { human missions } \\
\text { simultaneously. }\end{array}$ & $\begin{array}{l}\text { Essential for providing communication routes for many spacecraft simultaneously so that many data } \\
\text { streams can be routed from end-to-end autonomously. }\end{array}$ \\
\hline Multiple quality of service levels & QoS diversity is required to handle voice, video, science data and control data simultaneously. \\
\hline $\begin{array}{l}\text { Minimum latency within the } \\
\text { networks. }\end{array}$ & $\begin{array}{l}\text { Required for maintaining the tightest possible control loops that are necessary in most human-operated } \\
\text { remote missions. It also helps for keeping human-human communications as close to real-time as } \\
\text { possible. }\end{array}$ \\
\hline $\begin{array}{l}\text { Provide navigation capabilities } \\
\text { within telemetry signals. }\end{array}$ & Needed for missions that must coordinate their activities and for flying in formations. \\
\hline $\begin{array}{l}\text { Operate in extreme } \\
\text { environments }\end{array}$ & $\begin{array}{l}\text { In-space hardware must survive solar flares and cold temperatures. Planetary/moon hardware faces } \\
\text { large temperature swings (Moon, Mars), high radiation (Europa), high temperature (Mercury). }\end{array}$ \\
\hline
\end{tabular}

NASA's communication infrastructure will become an autonomously operated system of networks on the ground and in-space. It will be possible for an in-space human or robotic spacecraft, rover, or ground-based user to demand and receive access to an arm of the network from nearly anywhere on or around the Earth, the Moon, or the Solar System. An integrated architecture that implements an infrastructure with the desired characteristics is made up of several regions of interest where groups of communication nodes represented by science and human missions will likely need access to modern networked, high data rate communications for conveying images, science data, voice, video, and control data among themselves and with Earth. The nodal regions of interest include the Earth vicinity from its surface to high Earth orbits, the Moon vicinity from lunar surface to the near and far Earth-Moon Lagrangian halo orbits $\left(\mathrm{EM}_{\mathrm{L} 1}\right.$ and $\left.\mathrm{EM}_{\mathrm{L} 2}\right)$, the Earth-Sun Lagrangian orbits $\left(\mathrm{ES}_{\mathrm{L} 1}, \mathrm{ES}_{\mathrm{L} 2}, \mathrm{ES}_{\mathrm{L} 4}, \mathrm{ES}_{\mathrm{L} 5}\right)^{8}$, Mars vicinity 
from its surface to the Mars synchronous orbit, Jupiter vicinity from its atmosphere to its Jupiter-Sun Lagrangian orbits $\left(\mathrm{JS}_{\mathrm{L} 1}, \mathrm{JS}_{\mathrm{L} 2}\right)$, and the neighborhoods of the rest of the planets, moons and objects in the Solar System. In this paper we cover only the Earth-Sun Lagrangian orbits at $\mathrm{ES}_{\mathrm{L} 4}$ and $\mathrm{ES}_{\mathrm{L} 5}$ insofar as they may be used in support of missions to Mars. We do not cover the nodal regions beyond Mars. The architectural scenario described in the following sections implements the evolutionary space communications architecture, its architectural elements and interfaces, the science it supports, and its concept of operations.

\section{A. Earth Vicinity Communications Infrastructure}

The Earth vicinity communications infrastructure for observation and exploration missions is diagrammed in Fig. 3 and includes the LEO, MEO, GEO, HEO relay satellites that may be implemented.

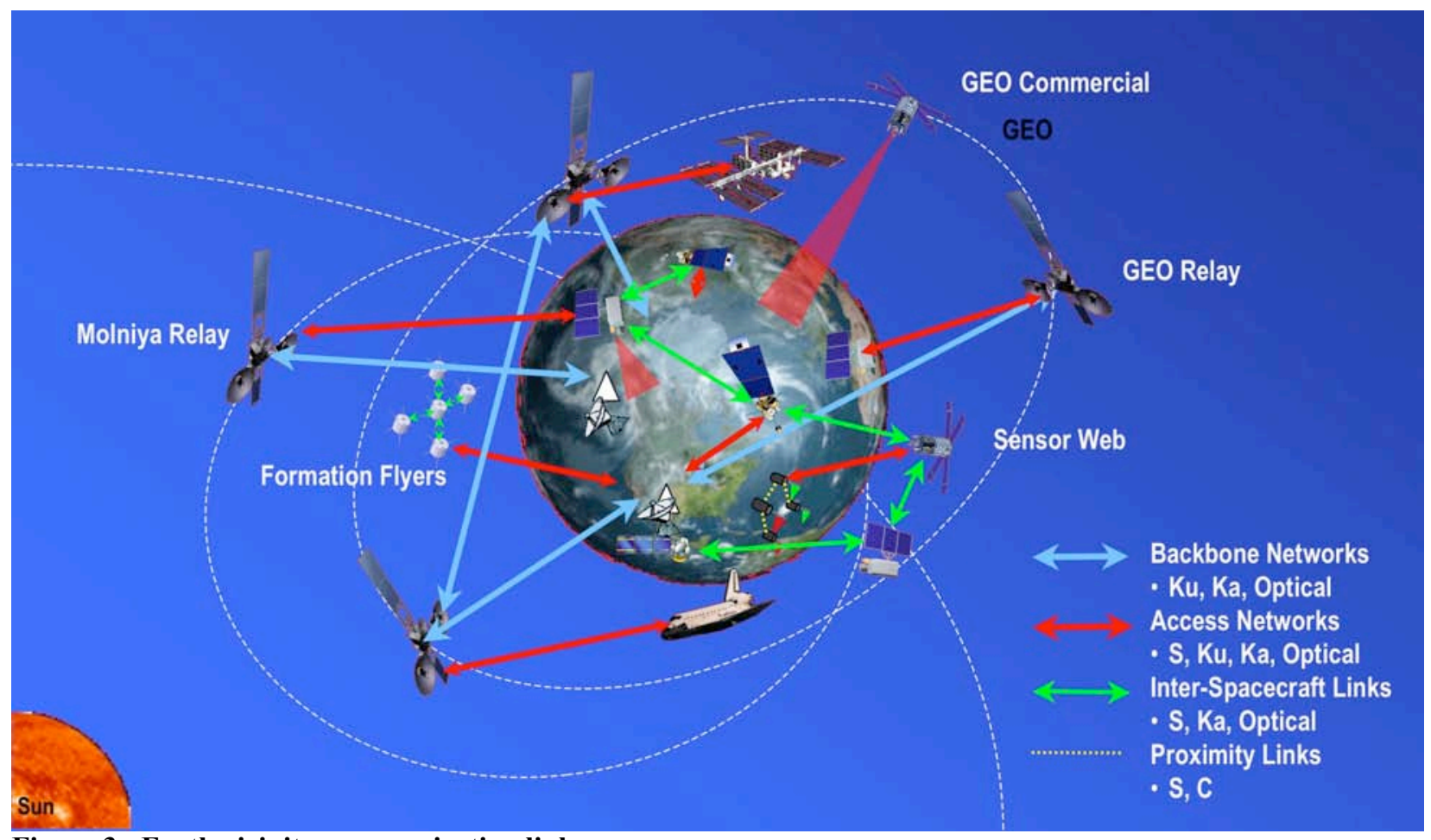

Figure 3: Earth vicinity communication links.

A listing of all of the optional node-to-node links that were considered, their data rates, link distances, likely technologies, and types of service is given in Table 3. The node-to-node links considered in the table include:

1) from TDRSS (or similar) geosynchronous space network links to Earth observing satellites and human missions;

2) from Medium Earth Orbit (MEO) relay networks links to Earth observing satellites and human missions;

3) from High Earth Orbit (HEO) including Molniya orbit relay networks to Earth observing satellites and human missions;

4) from Earth orbiting missions to HEO, GEO, or MEO networks;

5) from Low Earth Orbit (LEO) satellite to ground terminals;

6) between LEO satellites configured into a Sensor Web;

7) between ISS or Shuttle and HEO, GEO, or MEO networks; and

8) between ground terminals and ISS or Shuttle. 
Table 3: Characteristics and requirements of node-to-node link options that were considered for Earth vicinity communications.

\begin{tabular}{|c|c|c|c|c|}
\hline Node-to Node Link & $\begin{array}{l}\text { Data Rate } \\
\text { (Mbps) }\end{array}$ & Distance & Technology & Service \\
\hline \multicolumn{5}{|c|}{ 1) Space network (similar to TDRSS) element link to: } \\
\hline NASA LEO satellite & 1,200 & $35,000 \mathrm{~km}$ & Optical & Demand access data IP network services \\
\hline NASA LEO satellite low rate & 10 & $35,000 \mathrm{~km}$ & X-band & $\begin{array}{l}\text { Multiple access on-demand to move data, } \\
\text { emergency, TT\&C }\end{array}$ \\
\hline Human spacecraft & 1,200 & $35,000 \mathrm{~km}$ & Optical & $\begin{array}{l}\text { Bidirectional voice, video, data access data } \\
\text { services }\end{array}$ \\
\hline Space network element (crosslink) & 10,000 & $35,000 \mathrm{~km}$ & Optical & Bidirectional backbone data \\
\hline Lunar missions & 1,000 & $0.25 \mathrm{Mkm}$ & Optical & Bidirectional voice, HDTV, data \\
\hline Earth-Sun L1, L2 & 300 & $1.5 \mathrm{Mkm}$ & Optical & Backbone and Science data \\
\hline Mars missions & 100 & $2.5 \mathrm{AU}$ & Optical & Bidirectional voice, HDTV, data \\
\hline \multicolumn{5}{|l|}{ 2) MEO relay link to: } \\
\hline NASA LEO satellite & 1,000 & $4,000 \mathrm{~km}$ & Optical & Demand access data IP network services \\
\hline NASA LEO satellite low rate & 10 & $4,000 \mathrm{~km}$ & Ka-, X-band & $\begin{array}{l}\text { Multiple access on-demand to move data, } \\
\text { emergency, TT\&C }\end{array}$ \\
\hline Human spacecraft & 1,000 & $4,000 \mathrm{~km}$ & Optical & $\begin{array}{l}\text { Bidirectional voice, video, data access data } \\
\text { services }\end{array}$ \\
\hline MEO network element (crosslink) & 10,000 & $4,000 \mathrm{~km}$ & Optical & Bidirectional backbone data \\
\hline \multicolumn{5}{|l|}{ 3) HEO and Molniya relay link to: } \\
\hline NASA LEO satellite & 500 & $100,000 \mathrm{~km}$ & Optical & Demand access data IP network services \\
\hline NASA LEO satellite low rate & 5 & $100,000 \mathrm{~km}$ & Ka-, X-band & $\begin{array}{l}\text { Multiple access on-demand to move data, } \\
\text { emergency, TT\&C }\end{array}$ \\
\hline Human spacecraft & 500 & $100,000 \mathrm{~km}$ & Optical & $\begin{array}{l}\text { Bidirectional voice video, data access data } \\
\text { services }\end{array}$ \\
\hline \multicolumn{5}{|l|}{ 4) Earth satellite link to: } \\
\hline NASA HEO satellite & 500 & $100,000 \mathrm{~km}$ & Optical & Demand access data IP network services \\
\hline NASA HEO satellite low rate & 5 & $100,000 \mathrm{~km}$ & Ka-, X-band & $\begin{array}{l}\text { Multiple access on-demand to move data, } \\
\text { emergency, TT\&C }\end{array}$ \\
\hline NASA GEO satellite & 1,200 & $35,000 \mathrm{~km}$ & Optical & Demand access data IP network services \\
\hline NASA GEO satellite low rate & 10 & $35,000 \mathrm{~km}$ & Ka-, X-band & $\begin{array}{l}\text { Multiple access on-demand to move data, } \\
\text { emergency, TT\&C }\end{array}$ \\
\hline NASA MEO satellite & 1,000 & $4,000 \mathrm{~km}$ & Optical & Demand access data IP network services \\
\hline NASA MEO satellite low rate & 10 & $4,000 \mathrm{~km}$ & Ka-, X-band & $\begin{array}{l}\text { Multiple access on-demand to move data, } \\
\text { emergency, TT\&C }\end{array}$ \\
\hline \multicolumn{5}{|l|}{ 5) Ground terminal link to: } \\
\hline NASA LEO satellite & $>1,000$ & $2,000 \mathrm{~km}$ & Ka-band & Access data services \\
\hline \multicolumn{5}{|l|}{ 6) LEO satellite link to: } \\
\hline NASA LEO satellite & 1,000 & 4,000 & Ka-band & Share and route data \\
\hline \multicolumn{5}{|l|}{ 7) ISS or Shuttle link to: } \\
\hline NASA HEO satellite & 500 & $100,000 \mathrm{~km}$ & Optical & Demand access data IP network services \\
\hline NASA HEO satellite low rate & 5 & $100,000 \mathrm{~km}$ & Ka-, X-band & $\begin{array}{l}\text { Multiple access on-demand to move data, } \\
\text { emergency, TT\&C }\end{array}$ \\
\hline NASA GEO satellite & 1,200 & $35,000 \mathrm{~km}$ & Optical & Demand access data IP network services \\
\hline NASA GEO satellite low rate & 10 & $35,000 \mathrm{~km}$ & Ka-, X-band & $\begin{array}{l}\text { Multiple access on-demand to move data, } \\
\text { emergency, TT\&C }\end{array}$ \\
\hline NASA MEO satellite & 1,000 & $4,000 \mathrm{~km}$ & Optical & Demand access data IP network services \\
\hline NASA MEO satellite low rate & 10 & $4,000 \mathrm{~km}$ & Ka-, X-band & $\begin{array}{l}\text { Multiple access on-demand to move data, } \\
\text { emergency, TT\&C }\end{array}$ \\
\hline \multicolumn{5}{|l|}{ 8) Ground terminal link to: } \\
\hline ISS or Shuttle & $>1,000$ & $2,000 \mathrm{~km}$ & Ka-band & Access data services \\
\hline
\end{tabular}




\section{B. Lunar Communication Infrastructure}

The Moon vicinity communications infrastructure for robotic and human missions diagrammed in Fig. 4 includes Earth-Moon Lagrangian halo orbit relay satellites at $\mathrm{EM}_{\mathrm{L} 1}$ and $\mathrm{EM}_{\mathrm{L} 2}$, lunar orbit relay satellites, and lunar surface wireless local area networks (WLANs) that may be implemented.

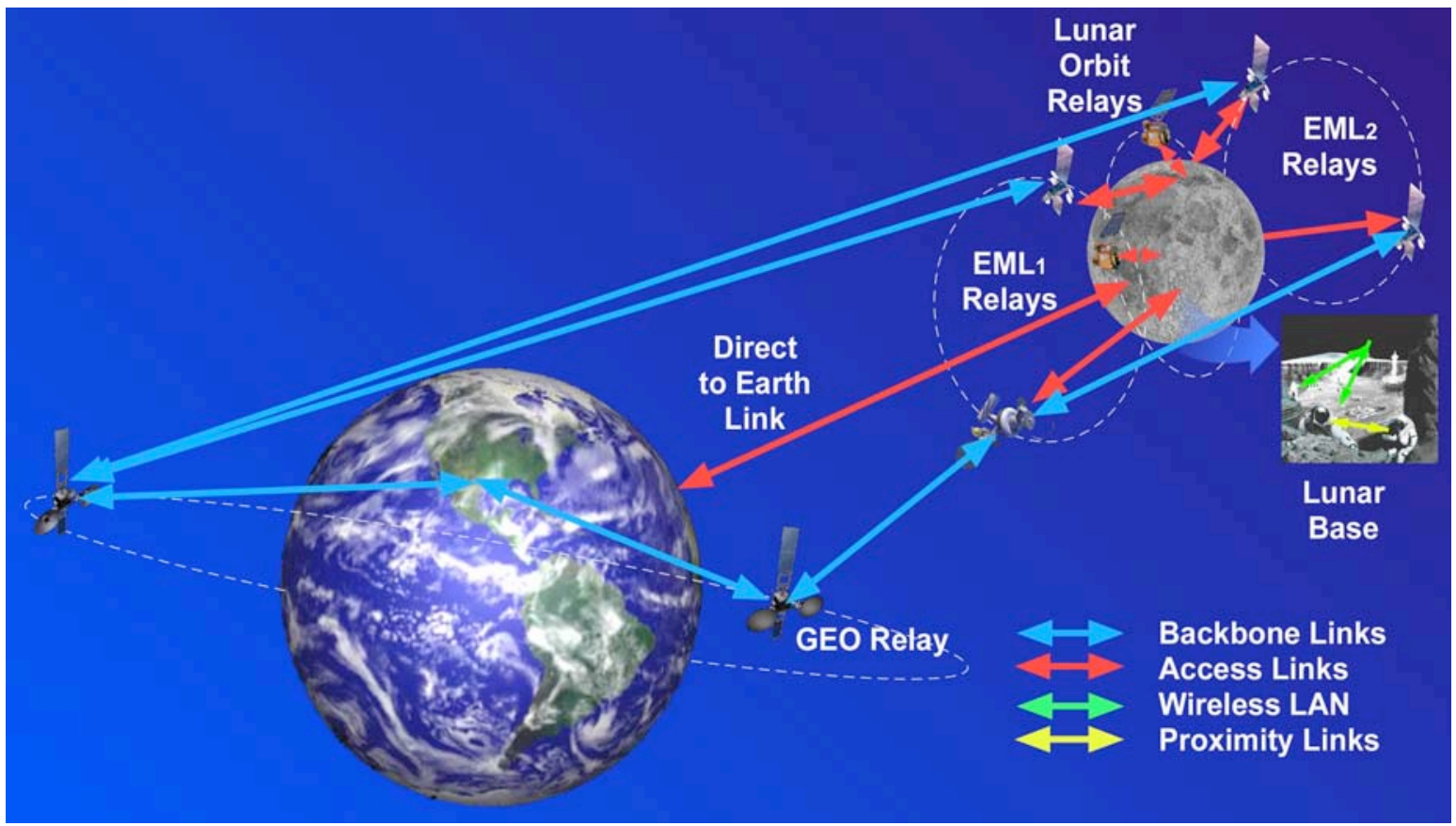

Figure 4: Moon vicinity and Earth-Moon communication links.

Table 4 is a list of the optional Earth to Lunar vicinity node-to-node communication links and the links between entities on the Lunar surface or in Lunar orbit that were considered for supporting robotic and human missions to the Moon. The options that were considered are grouped in the table as follows:

1) Large Satellites in Medium Moon Orbit (LSMMO) - A constellation of 8 communications relays are placed in two orbital planes ( 3 active and 1 spare in each plane) that are 90 degrees out of phase, one polar and one equatorial orbit at $>2000 \mathrm{~km}$ altitude to provide nearly 24/7 coverage between Earth and missions anywhere on the lunar surface and between entities on that surface. Data from lunar surface to lunar surface are also routed around the constellation. Each satellite has a link to Earth, to the lunar surface, and a satellite-tosatellite crosslink package.

2) Communication relay spacecraft placed in Earth-Moon L1 $\left(E_{\mathrm{L} 1}\right)$ halo orbit - Two spacecraft on opposite sides of the $\mathrm{EM}_{\mathrm{L} 1}$ halo orbit can provide nearly $100 \% 24 / 7$ coverage of most of the Earth-facing side of the Moon. A large halo orbit diameter with three or four relay spacecraft would provide 24/7 coverage of the rim of the Moon. Communication between near side Moon surface entities via an $\mathrm{EM}_{\mathrm{L} 1}$ relay has moderate latency of $\approx 0.4 \mathrm{~s}$.

3) Communication relay spacecraft placed in Earth-Moon L2 $\left(\mathrm{EM}_{\mathrm{L} 2}\right)$ halo orbit - Two spacecraft on opposite sides of the $\mathrm{EM}_{\mathrm{L} 2}$ halo orbit can provide nearly $100 \% 24 / 7$ coverage of most of the far side of the Moon. A large halo orbit diameter with three or four relay spacecraft would provide 24/7 coverage of the rim of the Moon from the far side. Communication between far side Moon surface entities via an $\mathrm{EM}_{\mathrm{L} 2}$ relay has moderate latency of $\approx 0.4 \mathrm{~s}$. Communication from the far side Moon surface to an L2 satellite to an L1 satellite and then to the near side Moon surface has latency of $\approx 1 \mathrm{~s}$.

4) Small Satellites in Low Moon Orbit (SSLMO) - A constellation of 35 small communications relays are placed in 5 equally spaced low orbit orbit planes with 6 active and 1 spare satellite in each plane to provide nearly 24/7 coverage between Earth and missions anywhere on the lunar surface and between entities on that surface. Data from lunar surface to lunar surface are also routed around the constellation. Each satellite has 2 to 4 communication packages for communication to the lunar surface and for inter-satellite crosslinks. 
Table 4: Characteristics and requirements of node-to-node link options that were considered for Earth to Moon and Moon vicinity communications.

\begin{tabular}{|c|c|c|c|c|}
\hline Node-to Node Link & $\begin{array}{l}\text { Data Rate } \\
\text { (Mbps) }\end{array}$ & Distance & Technology & Service \\
\hline \multicolumn{5}{|l|}{ 1) LSMMO relay spacecraft constellation } \\
\hline Earth ground & $>300$ & $384,000 \mathrm{~km}$ & Ka-, X-bands & Backbone data services \\
\hline Earth orbit relay & 1,000 & $384,000 \mathrm{~km}$ & Optical & Backbone data services \\
\hline LSMMO relay spacecraft (crosslink) & 1,000 & $6,500 \mathrm{~km}$ & Optical, Ka & Backbone data services \\
\hline Moon low rate & 10 & $2,700 \mathrm{~km}$ & Ka-, X-bands & Emergency, TT\&C \\
\hline Moon science orbiter & 100 & $2,700 \mathrm{~km}$ & Ka-, X-bands & Science files \\
\hline Moon human outpost & 1,000 & $2,700 \mathrm{~km}$ & Optical, Ka & Bidirectional voice, HDTV, data \\
\hline \multicolumn{5}{|c|}{ 2) Earth-Moon L1 (EML1) communication relay spacecraft } \\
\hline Earth ground & $>300$ & $323,000 \mathrm{~km}$ & Ka-, X-bands & Backbone data services \\
\hline Earth orbit relay & 1,000 & $323,000 \mathrm{~km}$ & Optical & Backbone data services \\
\hline Earth-Moon L1 Gateway & 1,000 & $10,000 \mathrm{~km}$ & Optical, Ka & Access data services \\
\hline Moon relays, high rate & 1,000 & $61,000 \mathrm{~km}$ & Optical, Ka & Backbone data services \\
\hline Moon low rate & 10 & $61,000 \mathrm{~km}$ & Ka-, X-bands & Emergency, TT\&C \\
\hline Moon science orbiter & 100 & $61,000 \mathrm{~km}$ & Ka-, X-bands & Science files \\
\hline Moon human outpost & 1,000 & $61,000 \mathrm{~km}$ & Optical, Ka & Bidirectional voice, HDTV, data \\
\hline \multicolumn{5}{|c|}{ 3) Earth-Moon L2 (EML2) communication relay spacecraft } \\
\hline Earth ground & $>300$ & $445,000 \mathrm{~km}$ & Ka-, X-bands & Backbone data services \\
\hline Earth orbit relay & 1,000 & $445,000 \mathrm{~km}$ & Optical & Backbone data services \\
\hline Moon relays, high rate & 1,000 & $61,000 \mathrm{~km}$ & Optical, Ka & Backbone data services \\
\hline Moon low rate & 10 & $61,000 \mathrm{~km}$ & Ka-, X-bands & Emergency, TT\&C \\
\hline Moon science orbiter & 100 & $61,000 \mathrm{~km}$ & Ka-, X-bands & Science files \\
\hline Moon human outpost & 1,000 & $61,000 \mathrm{~km}$ & Optical, Ka & Bidirectional voice, HDTV, data \\
\hline \multicolumn{5}{|c|}{ 4) Small Satellite, Low Moon Orbit (SSLMO) relay spacecraft constellation } \\
\hline SSLMO relay spacecraft (crosslink) & 1,000 & $2,100 \mathrm{~km}$ & $\mathrm{Ka}$ & Backbone data services \\
\hline Moon low rate & 10 & $650 \mathrm{~km}$ & Ka-, X-bands & Emergency, TT\&C \\
\hline Moon science orbiter & 100 & $650 \mathrm{~km}$ & Ka-, X-bands & Science files \\
\hline Moon human outpost & 1,000 & $650 \mathrm{~km}$ & $\mathrm{Ka}$ & Bidirectional voice, HDTV, data \\
\hline \multicolumn{5}{|c|}{ 5) Small Satellite, Low Moon Orbit (SSLMO) Lunar surface terminal relays } \\
\hline Earth ground & $>300$ & $384,000 \mathrm{~km}$ & $\mathrm{Ka}$ & Backbone data services \\
\hline Earth orbit relay & 1,000 & $384,000 \mathrm{~km}$ & Optical & Backbone data services \\
\hline SSLMO relay spacecraft (crosslink) & 1,000 & $650 \mathrm{~km}$ & $\mathrm{Ka}$ & Backbone data services \\
\hline \multicolumn{5}{|c|}{ 6) Human lunar outpost sends and receives voice, video, and data using direct to Earth links } \\
\hline SSLMO relay & 1,000 & $650 \mathrm{~km}$ & $\mathrm{Ka}$ & Bidirectional voice, HDTV, data \\
\hline LSMMO relay & 1,000 & $2,700 \mathrm{~km}$ & Optical, Ka & Bidirectional voice, HDTV, data \\
\hline Earth-Moon L1 relay & 1,000 & $323,110 \mathrm{~km}$ & Optical, Ka & $\begin{array}{l}\text { Bidirectional, multipoint, voice, video, } \\
\text { remote control, science data, emergency }\end{array}$ \\
\hline Earth orbit relays & 1,000 & $384,400 \mathrm{~km}$ & Optical, Ka & $\begin{array}{l}\text { Bidirectional, multipoint, voice, video, } \\
\text { remote control, science data, emergency }\end{array}$ \\
\hline Earth terminal & 200 & $384,400 \mathrm{~km}$ & Ka-, X-bands & Science data, emergency, TT\&C \\
\hline \multicolumn{5}{|c|}{ 7) Lunar outpost wireless local area network (WLAN) } \\
\hline Other lunar surface entity at close range & $>100$ & $100 \mathrm{~m}$ & $\begin{array}{l}\text { Ka-, } \mathrm{X}-, \mathrm{C}- \\
\text { bands }\end{array}$ & $\begin{array}{l}\text { Bidirectional, multipoint, voice, video, } \\
\text { remote control, data, emergency }\end{array}$ \\
\hline $\begin{array}{l}\text { Other lunar surface entity at long surface } \\
\text { distance }\end{array}$ & $>50$ & $50 \mathrm{~km}$ & $\begin{array}{l}\text { Ka-, } \mathrm{X}-, \mathrm{C}- \\
\text { bands }\end{array}$ & $\begin{array}{l}\text { Bidirectional, multipoint, voice, video, } \\
\text { remote control, data, emergency }\end{array}$ \\
\hline
\end{tabular}

5) Lunar surface terminal relays SSLMO communication relays - Since the satellites were assumed to be small and inexpensive, it was not expected that they would have high data rate capabilities to Earth. Consequently two high data rate lunar surface terminals were assumed to be emplaced on the near-side of the Moon with high data rate capabilities with Earth. The SSLMO satellites would then route data from a surface entity, around the Moon, and down to a lunar surface terminal that routes the data to Earth. 
6) Human lunar outpost communication links - Links are to Earth via SSLMO relays, LSMMO relays, EML1 relays, relays in Earth orbit, or direct-to-Earth terminals for sending and receiving voice, video, and data. There is a two-way latency of $2.5 \mathrm{~s}$ in signal turnaround with earth.

7) Lunar outpost wireless local area networks (WLAN) - Characteristics of surface communication networks between robots and humans include omnidirectional, local communications over short ranges $(\approx 100 \mathrm{~m})$ and directional, (antenna pointed) communications over longer local distances $(\approx 50 \mathrm{~km})$ capable of handling voice, video, control, and data passing between multiple local entities.

\section{Earth-Mars and Mars Vicinity Communications}

The Mars vicinity communications infrastructure for robotic and human missions is diagrammed in Fig. 5 along with the deep space communications. It includes Mars communication relay satellites, science spacecraft, atmospheric craft, surface rovers, landers, sensor, and human outposts that may be implemented at Mars.

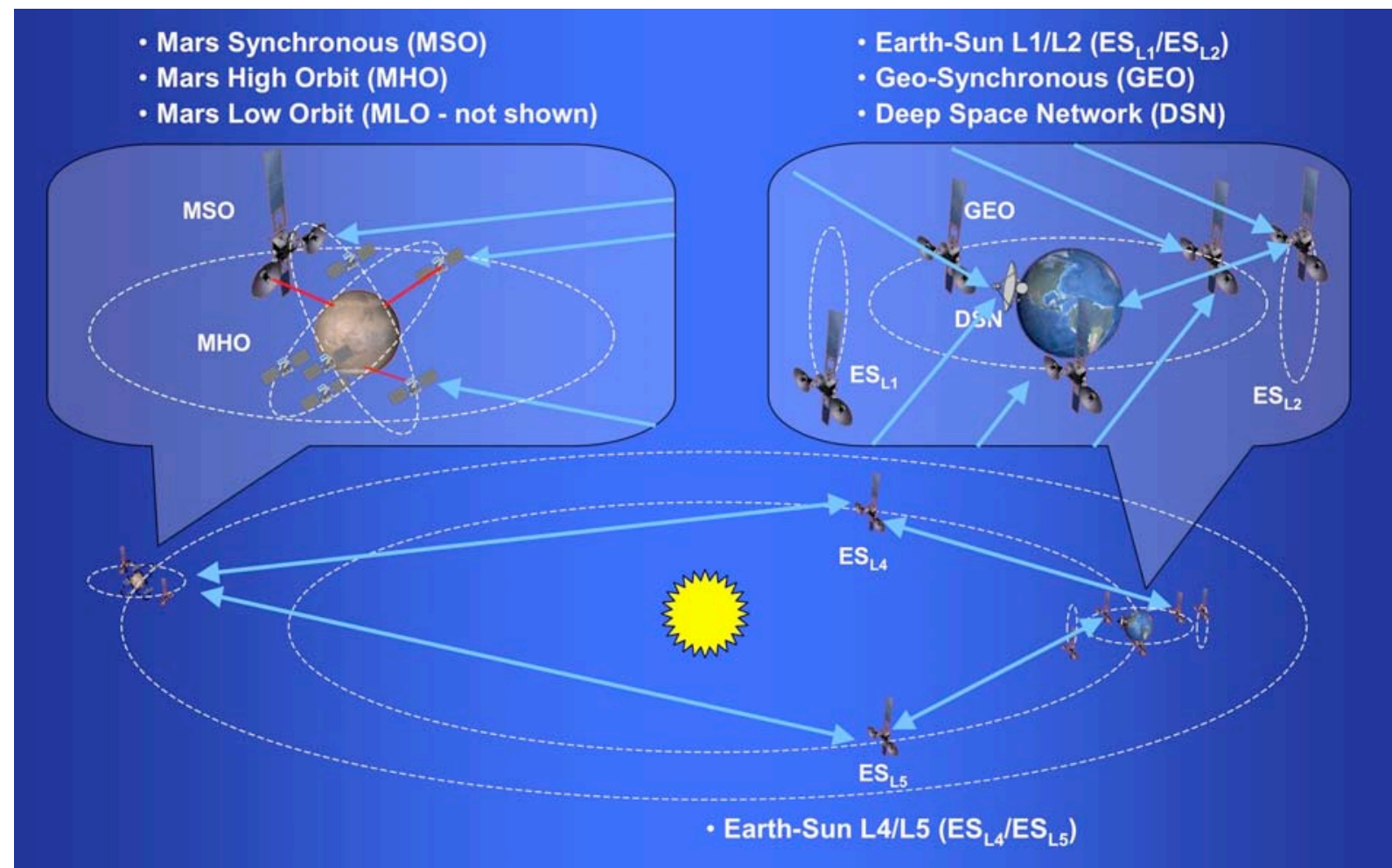

Figure 5: Mars vicinity and Earth-Mars communication links.

The node-to-node links between Earth and Mars and at Mars are identified in Table 5 as follows:

1) Earth-Mars communication using relay spacecraft placed at Earth-Sun L4 ( $\mathrm{ESL}_{4}$, Earth-leading orbit) and/or Earth-Sun L5 (ESL 5 , trailing orbit) - A relay at either of these locations enables communication around the Sun during times of solar conjunction (when the Sun blocks the Earth view of Mars). Relay stations at both L4 and L5 add redundancy to the communication paths around the Sun.

2) Earth-Mars communication using Mars Synchronous Orbit (MSO) communications relay satellite - A relay satellite placed in MSO can provide 24/7 coverage between Earth and one side of Mars. Three MSO satellites would be needed to provide full coverage between the Earth and Mars.

3) Earth-Mars communication using Mars High Orbit (MHO) communications relay satellite network - Four to six communication relay satellites with crosslinks placed in MHO can provide 24/7 coverage of the entire planet.

4) Earth-Mars communication - Mars Low Orbit (MLO) science satellite with add-on relay network functions are already being deployed around Mars. These satellites are capable of low data rate communications $(<1 \mathrm{Mbps})$.

5) Mars vicinity communications - Data are sent and commands received from Mars orbit, atmosphere, or surface human or robotic entity using relays in MSO, MHO, and MLO. Direct to Earth communications are also considered. 
6) Mars outpost wireless local area network (WLAN) - Characteristics of surface communication networks between robots and humans include omnidirectional, local communications over short ranges $(\approx 100 \mathrm{~m})$ and directional, (antenna pointed) communications over longer local distances $(\approx 50 \mathrm{~km})$ capable of handling voice, video, control, and data passing between multiple local entities.

Table 5: Characteristics and requirements of node-to-node link options that were considered for Earth to Mars and Mars vicinity communications.

\begin{tabular}{|c|c|c|c|c|}
\hline Node-to Node Link & $\begin{array}{l}\text { Data Rate } \\
\text { (Mbps) }\end{array}$ & Distance & Technology & Service \\
\hline \multicolumn{5}{|l|}{ 1) Earth-Sun L3, L4 Relay Link to: } \\
\hline Earth ground & $>100$ & $1 \mathrm{AU}$ & Optical & Backbone data services \\
\hline Earth orbit relay & $>100$ & $1 \mathrm{AU}$ & Optical & Backbone data services \\
\hline Mars relays, high rate & 100 & $2.5 \mathrm{AU}$ & Optical & Backbone data services \\
\hline Mars low rate & 1 & $2.5 \mathrm{AU}$ & Ka-, X-bands & Emergency, TT\&C \\
\hline Mars science S/C & 10 & $2.5 \mathrm{AU}$ & Optical, Ka & Science files \\
\hline Mars human outpost & 100 & $2.5 \mathrm{AU}$ & Optical, Ka & Bidirectional voice, HDTV, data \\
\hline \multicolumn{5}{|l|}{ 2) MSO Relay Link to: } \\
\hline Earth ground & $>1$ & $2.5 \mathrm{AU}$ & Ka-, X-band & Emergency, TT\&C \\
\hline Earth L1, L2, L4, L5, GEO orbit relay & $>100$ & $2.5 \mathrm{AU}$ & Optical & Bidirectional Backbone data \\
\hline Mars low rate & 1 & $10,000 \mathrm{~km}$ & Ka-, X-bands & Emergency, TT\&C \\
\hline Mars science orbiters & 100 & $10,000 \mathrm{~km}$ & Optical, Ka & Multiple science S/C files \\
\hline Mars surface robots & 10 & $10,000 \mathrm{~km}$ & $\mathrm{Ka}$ & Multiple science S/C files \\
\hline Mars human outpost & 100 & $10,000 \mathrm{~km}$ & Optical, Ka & Bidirectional voice, HDTV, data \\
\hline \multicolumn{5}{|l|}{ 3) MHO Relay Link to: } \\
\hline Earth ground & $>1$ & $2.5 \mathrm{AU}$ & Ka-, X-band & Emergency, TT\&C \\
\hline Earth L1, L2, L4, L5, GEO orbit relay & $>100$ & $2.5 \mathrm{AU}$ & Optical & Bidirectional Backbone data \\
\hline Mars low rate & 1 & $4,000 \mathrm{~km}$ & Ka-, X-bands & Emergency, TT\&C \\
\hline Mars science orbiters & 100 & $4,000 \mathrm{~km}$ & Optical, Ka & Multiple science S/C files \\
\hline Mars surface robots & 10 & $4,000 \mathrm{~km}$ & $\mathrm{Ka}$ & Multiple science S/C files \\
\hline Mars human outpost & 100 & $4,000 \mathrm{~km}$ & Optical, Ka & Bidirectional voice, HDTV, data \\
\hline \multicolumn{5}{|c|}{ 4) MLO Relay Package Onboard Science Spacecraft Link to: } \\
\hline Earth ground & $>1$ & $2.5 \mathrm{AU}$ & Ka-, X-band & Emergency, TT\&C \\
\hline Earth L1, L2, L4, L5, GEO orbit relay & $>10$ & $2.5 \mathrm{AU}$ & Ka-band & Bidirectional Backbone data \\
\hline Mars low rate & 1 & $400 \mathrm{~km}$ & Ka-, X-bands & Emergency, TT\&C \\
\hline Mars science orbiters & 10 & $400 \mathrm{~km}$ & Ka-, X-bands & Multiple science S/C files \\
\hline Mars surface robots & 10 & $400 \mathrm{~km}$ & $\mathrm{Ka}, \mathrm{X}$-band & Multiple science S/C files \\
\hline Mars human outpost & 10 & $400 \mathrm{~km}$ & Ka-band & Bidirectional voice, HDTV, data \\
\hline \multicolumn{5}{|c|}{ 5) Mars orbit, atmosphere, or surface human or robotic entity link to: } \\
\hline MSO low rate & 1 & $10,000 \mathrm{~km}$ & Ka-, X-bands & Emergency, TT\&C \\
\hline MSO high rate & 100 & $10,000 \mathrm{~km}$ & Optical, Ka & Multiple science S/C files \\
\hline MHO low rate & 1 & $4,000 \mathrm{~km}$ & Ka-, X-bands & Emergency, TT\&C \\
\hline MHO high rate & 100 & $4,000 \mathrm{~km}$ & Optical, Ka & Multiple science S/C files \\
\hline MLO low rate & 1 & $1000 \mathrm{~km}$ & Ka-, X-bands & Emergency, TT\&C \\
\hline MLO high rate & 10 & $1000 \mathrm{~km}$ & $\mathrm{Ka}$ & Multiple science S/C files \\
\hline Earth terminal & 0.1 & $2.5 \mathrm{AU}$ & Ka-, X-bands & Emergency, TT\&C \\
\hline Earth orbit relays & 10 & $2.5 \mathrm{AU}$ & $\mathrm{Ka}$ & Multiple science S/C files \\
\hline \multicolumn{5}{|c|}{ 6) Mars outpost wireless local area network (WLAN) link to: } \\
\hline Mars surface/atmosphere entity & $>100$ & $100 \mathrm{~m}$ & $\begin{array}{l}\text { Ka-, X-, C- } \\
\text { bands }\end{array}$ & $\begin{array}{l}\text { Bidirectional, multipoint, voice, video, } \\
\text { remote control, data, emergency. }\end{array}$ \\
\hline Mars surface/atmosphere entity & $>50$ & $50 \mathrm{~km}$ & $\begin{array}{l}\text { Ka-, } \mathrm{X}-, \mathrm{C}- \\
\text { bands }\end{array}$ & $\begin{array}{l}\text { Bidirectional, multipoint, voice, video, } \\
\text { remote control, data, emergency. }\end{array}$ \\
\hline
\end{tabular}

\section{Conclusions}

In this paper, we have described a space communication architecture that can meet the challenging requirements for human and robotic exploration missions to the Moon and that can evolve to enable and enhance human and robotic exploration missions to Mars. The systematic identification of the communications architectural elements and of the optional ways they can be implemented serves as valuable tool for indicating to the mission planner and 
scientist the possible communication capabilities that can be realized by the alternative configurations. It serves well for constructing strawman architectures for evaluating which options have the highest payback potential. Extensive system cost and risk analysis and trades will be the next logical step to refine the architecture for implementation.

\section{References}

${ }^{1}$ NASA, "The Vision for Space Exploration," February 2004.

${ }^{2}$ King, M. D., and Greenstone, R., Editors, 1999 EOS Reference Handbook, EOS Project Science Office, 1999, pp. 15-35.

${ }^{3}$ Tai, W. S., Deep Space Mission System (DSMS) Services Catalog, Version 7.5, Jet Propulsion Laboratory, DSMS No. 820100, 2003, pp. 1-1

${ }^{4}$ Bhasin, K., and Hayden, J. L., "Space Internet Architectures and Technologies for NASA Enterprises," Int. J. Satell. Commun. 2002; 20, 311-332.

${ }^{5}$ Bhasin, K., and Hayden, J. L., "Evolutionary Space Communications Architectures for Human/Robotic Exploration and Science Missions," Space Technology \& Applications International Forum, (STAIF-2004), 2004, Albuquerque, NM.

${ }^{6}$ Rash, J., Hogie, K., Casasanta, R., "Internet Technology for Future Space Missions," Proceedings of the International Telemetering Conference, San Diego, CA, 2002.

${ }^{7}$ Shames, P., “Architectural Models of Space Networking,” Space Internet Workshop, Cleveland, OH, June 2003.

${ }^{8}$ Bhasin, K., and Hayden, J. L., "Advanced communication architectures and technologies for missions to the outer planets," Forum On Innovative Approaches For Outer Planetary Exploration 2001-2020, Houston, TX, February 2001. 

Public reporting burden for this collection of information is estimated to average 1 hour per response, including the time for reviewing instructions, searching existing data sources, gathering and maintaining the data needed, and completing and reviewing the collection of information. Send comments regarding this burden estimate or any other aspect of this collection of information, including suggestions for reducing this burden, to Washington Headquarters Services, Directorate for Information Operations and Reports, 1215 Jefferson Davis Highway, Suite 1204, Arlington, VA 22202-4302, and to the Office of Management and Budget, Paperwork Reduction Project (0704-0188), Washington, DC 20503.

\begin{tabular}{|l|c|r|}
\hline 1. AGENCY USE ONLY (Leave blank) & 2. REPORT DATE & 3. REPORT TYPE AND DATES COVERED \\
July 2004 & Technical Memoran Mut
\end{tabular}

\section{TITLE AND SUBTITLE}

Developing Architectures and Technologies for an Evolvable NASA Space

Communication Infrastructure

\section{AUTHOR(S)}

Kul Bhasin and Jeffrey Hayden

WBS-22-302-20-2z

National Aeronautics and Space Administration

John H. Glenn Research Center at Lewis Field

Cleveland, Ohio 44135-3191

8. PERFORMING ORGANIZATION REPORT NUMBER

E-14583

\section{SPONSORING/MONITORING AGENCY NAME(S) AND ADDRESS(ES)}

National Aeronautics and Space Administration

Washington, DC 20546-0001

10. SPONSORING/MONITORING AGENCY REPORT NUMBER

NASA TM-2004-213108

AIAA-2004-3253

\section{SUPPLEMENTARY NOTES}

Prepared for the 22nd International Communications Satellite Systems Conference and Exhibit 2004 (ICSSC) sponsored by the American Institute of Aeronautics and Astronautics, Monterey, California, May 9-12, 2004. Kul Bhasin, NASA Glenn Research Center; and Jeffrey Hayden, Infinite Global Infrastructues LLC, West Chicago, Illinois 60185. Responsible person, Kul Bhasin, organization code 6100, 216-433-3676.

12a. DISTRIBUTION/AVAILABILITY STATEMENT

12b. DISTRIBUTION CODE

Unclassified - Unlimited

Subject Category: 32

Distribution: Nonstandard

Available electronically at http://gltrs.grc.nasa.gov

This publication is available from the NASA Center for AeroSpace Information, 301-621-0390.

13. ABSTRACT (Maximum 200 words)

Space communications architecture concepts play a key role in the development and deployment of NASA's future exploration and science missions. Once a mission is deployed, the communication link to the user needs to provide maximum information delivery and flexibility to handle the expected large and complex data sets and to enable direct interaction with the spacecraft and experiments. In human and robotic missions, communication systems need to offer maximum reliability with robust two-way links for software uploads and virtual interactions. Identifying the capabilities to cost effectively meet the demanding space communication needs of 21 st century missions, proper formulation of the requirements for these missions, and identifying the early technology developments that will be needed can only be resolved with architecture design. This paper will describe the development of evolvable space communication architecture models and the technologies needed to support Earth sensor web and collaborative observation formation missions; robotic scientific missions for detailed investigation of planets, moons, and small bodies in the solar system; human missions for exploration of the Moon, Mars, Ganymede, Callisto, and asteroids; human settlements in space, on the Moon, and on Mars; and great in-space observatories for observing other star systems and the universe. The resulting architectures will enable the reliable, multipoint, high data rate capabilities needed on demand to provide continuous, maximum coverage of areas of concentrated activities, such as in the vicinity of outposts in-space, on the Moon or on Mars.

14. SUBJECT TERMS

15. NUMBER OF PAGES

Communications; Space communications; Satellite communication

17. SECURITY CLASSIFICATION OF REPORT

Unclassified

18. SECURITY CLASSIFICATION
OF THIS PAGE
Unclassified

Unclassified

19. SECURITY CLASSIFICATION
OF ABSTRACT
Unclassified

Unclassified 

